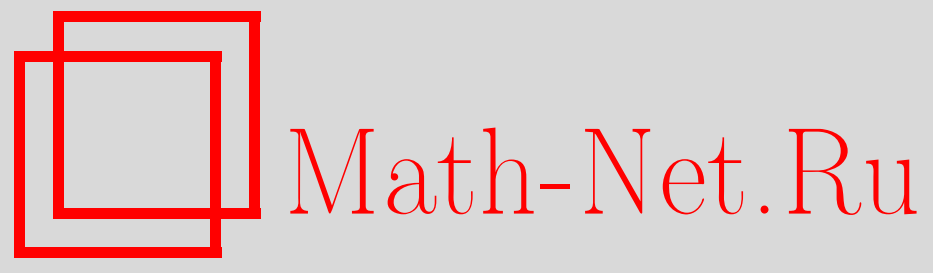

О. М. Сизова, Ультраметрическая диффузия в сильном центрально-симметричном поле, Вестн. Сам. гос. техн. ун-та. Сер. Физ.-мат. науки, 2015, номер 1, 87-104

DOI: https://doi.org/10.14498/vsgtu1389

Использование Общероссийского математического портала MathNet.Ru подразумевает, что вы прочитали и согласны с пользовательским соглашением

http://www . mathnet.ru/rus/agreement

Параметры загрузки:

IP : 54.224 .60 .19

26 апреля 2023 г., 15:55:35

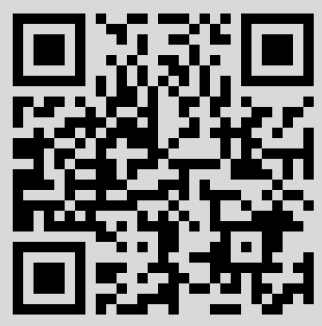


УДК $517.958+519.2$

\title{
УЛЬТРАМЕТРИЧЕСКАЯ ДИФФУЗИЯ В СИЛЬНОМ ЦЕНТРАЛЬНО-СИММЕТРИЧНОМ ПОЛЕ
}

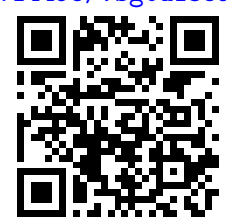

\section{О. М. Сизова}

Институт химической физики им. Н. Н. Семенова РАН, Россия, 119991, Москва, ул. Косыгина, 4.

\begin{abstract}
Аннотация
Рассмотрен случайный процесс на границе конечного регулярного дерева, помещённого во внешнее центрально-симметричное поле, относительно введённой ультраметрики. Показана процедура сведения этой задачи к задаче меньшей размерности. Также рассматривается случай предельно сильного поля, который удаётся решить аналитически. Приводятся решение для частного случая линейного роста иерархии барьеров переходов и оценка кинетики перехода в основное состояние.
\end{abstract}

Ключевые слова: ультраметрика, ультраметрическая диффузия, иерархический энергетический ландшафт.

doi: http://dx.doi.org/10.14498/vsgtu1389

Введение. В последние два десятилетия наблюдается рост интереса к случайным процессам над неархимедовыми полями. Если до начала 80-х годов эта тема звучала почти исключительно в математической литературе (см. $[2,3]$ и цитированную там литературу), то к настоящему моменту ультраметрические конструкции занимают видное место в физике конденсированных сред с беспорядком [4-6], динамике белковых молекул [7,8], математическом моделировании и дизайне молекулярных машин [9-12], описании компактной укладки ДНК в ядре клетки [13,14], и наконец, теории эволюции [15]. Складывается впечатление, что всякий раз, когда теория сталкивается с многомасштабной архитектурой, иерархической упорядоченностью и самоподобием, возникает желание использовать ультраметрическое пространство для описания состояний системы и её динамики. В таких случаях часто говорят о «сложных системах», управляющая функция которых (потенциальная или свободная энергия физических систем, или «ландшафт приспосабливаемости» эволюционных систем) представляет собой сильно пересечённую многомерную гиперповерхность с огромным числом локальных

(C) 2015 Самарский государственный технический университет.

\section{Образец для цитирования}

С изов а О. М. Ультраметрическая диффузия в сильном центрально-симметричном поле // Вестн. Сам. гос. техн. ун-та. Сер. Физ.-мат. науки, 2015. Т. 19, № 1. С. 87-104. doi: $10.14498 /$ vsgtu1389.

\section{Сведения об авторе}

Ольга Михайловна Сизова (olstet@mail.ru), научный сотрудник, отдел строения вещества.

*Настоящая статья представляет собой расширенный вариант доклада [1], сделанного автором на Четвёртой международной конференции «Математическая физика и её приложения» (Россия, Самара, 25 августа - 1 сентября 2014). 
экстремумов [16, 17]. Интересно, что обсуждение стохастической динамики на сильно пересечённых многомерных ландшафтах и попытки связать её с блужданиями на древообразных графах можно найти в работах генетиковэволюционистов 30-х годов прошедшего столетия (см., например, [18]), пытавшихся проложить путь от комбинаторной природы генетики к древообразному (таксономическому) описанию видового разнообразия. Новую волну интереса к этим идеям можно увидеть в работах середины 80-х годов прошедшего столетия, мотивированных желанием связать ультраметрическую картину фазовых состояний конденсированных систем с беспорядком с характерной для них аномальной (многомодовой) релаксационной кинетикой и использовать этот подход для описания динамики белка и кинетики ферментативной реакции [19-24]. К этому же периоду относится небольшая заметка [25], в которой была поставлена и решена задача об ультраметрическом случайном блуждании на дискретной решётке, оснащённой бинарным деревом ультраметрических расстояний. Эта заметка представляется удобной отправной точкой для постановки задачи настоящей статьи.

В работе [25] было рассмотрено случайное блуждание на границе регулярного бинарного дерева из $r$ уровней и $2^{r}$ листьев, занумерованных от 0 до $2^{r}-1$ (см. рис. 1), с заданной на них ультраметрикой. А именно, расстояние между любыми двумя листьями бинарного дерева (состояниями системы) определяется только номером $\gamma$ уровня, на котором находится вершина минимального поддерева, содержащего эти листья. При этом считается, что переход из одного состояния в другое происходит в результате преодоления барьера $H_{\gamma}$, высота которого есть функция ультраметрического расстояния между состояниями.

Обозначим за $f_{i}(t)$ переходную вероятность случайного блуждания на границе дерева, т. е. вероятность обнаружить блуждающую «частицу» в состоянии $i$ в момент времени $t$. Будем считать, что константа перехода через барьер $H_{\gamma}$ равна $e^{-H_{\gamma}}$, что физически согласуется с законом Аррениуса. Тогда, учитывая энтропийный фактор, определим константу перехода между двумя состояниями, разделёнными барьером $H_{\gamma}$, как

$$
q_{\gamma}=e^{-H_{\gamma}} / \mu(\gamma)
$$

где $\mu(\gamma)=2^{\gamma-1}-$ мера поддерева, в которое осуществляется переход через данный барьер.

Кинетическое уравнение, описывающее этот случайный процесс, имеет вид

$$
\frac{\partial \mathbf{f}(t)}{\partial t}=A \mathbf{f}(t)
$$

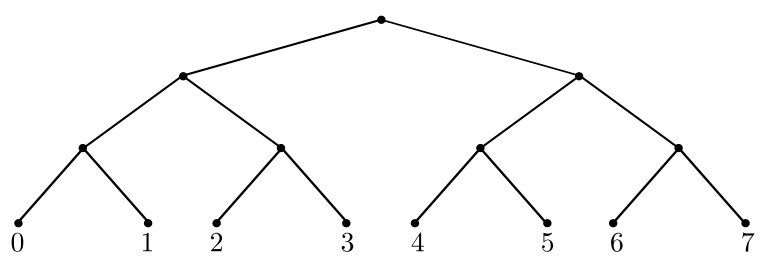

Рис. 1. Бинарное дерево при $r=3$

[Figure 1. Binary tree, when $r=3$ ] 
где $\mathbf{f}(t)=\left(f_{0}(t), \ldots, f_{2^{r}-1}(t)\right)$ и $A$ - матрица переходов вида

\begin{tabular}{|c|c|c|c|c|c|c|c|c|c|}
\hline \multirow{9}{*}{$A=$} & $d$ & $q_{1}$ & $q_{2}$ & $q_{2}$ & $q_{3}$ & $q_{3}$ & $q_{3}$ & $q_{3}$ & $\ldots$ \\
\hline & $q_{1}$ & $d$ & $q_{2}$ & $q_{2}$ & $q_{3}$ & $q_{3}$ & $q_{3}$ & $q_{3}$ & $\ldots$ \\
\hline & \multirow{2}{*}{$\begin{array}{l}q_{2} \\
q_{2}\end{array}$} & $q_{2}$ & $d$ & $q_{1}$ & $q_{3}$ & $q_{3}$ & $q_{3}$ & $q_{3}$ & $\cdots$ \\
\hline & & $q_{2}$ & $q_{1}$ & $d$ & $q_{3}$ & $q_{3}$ & $q_{3}$ & $q_{3}$ & $\cdots$ \\
\hline & \multirow{4}{*}{$\begin{array}{l}q_{3} \\
q_{3} \\
q_{3} \\
q_{3}\end{array}$} & $q_{3}$ & $q_{3}$ & $q_{3}$ & $d$ & $q_{1}$ & $q_{2}$ & $q_{2}$ & $\ldots$ \\
\hline & & $q_{3}$ & $q_{3}$ & $q_{3}$ & $q_{1}$ & $d$ & $q_{2}$ & $q_{2}$ & $\cdots$ \\
\hline & & $q_{3}$ & $q_{3}$ & $q_{3}$ & $q_{2}$ & $q_{2}$ & $d$ & $q_{1}$ & $\ldots$ \\
\hline & & $q_{3}$ & $q_{3}$ & $q_{3}$ & $q_{2}$ & $q_{2}$ & $q_{1}$ & $d$ & $\ldots$ \\
\hline & $:$ & : & : & : & : & $\vdots$ & : & $\vdots$ & $\ddots$ \\
\hline
\end{tabular}

где $d=-\left(q_{1}+2 q_{2}+2^{2} q_{3}+\cdots+2^{r-1} q_{r}\right)$ - сумма элементов по столбцу со знаком минус.

Заметим, что матрица (1) симметрична, вырождена и имеет блочно-иерархическую структуру. Как было показано в [25], собственные вектора такой матрицы не зависят от элементов $q_{\gamma}$. Более того они разбиваются на группы, образуя собственные подпространства $A_{\gamma}, \gamma=0, \ldots, r$ с собственными значениями $\lambda_{\gamma}$ следующего вида:

$$
\lambda_{0}=0: \quad A_{0}=\left\langle\left(\begin{array}{c}
1 \\
1 \\
\vdots \\
1
\end{array}\right)\right\rangle \text {; }
$$

$$
\begin{array}{r}
\lambda_{1}=-2\left(q_{1}+q_{2}+2 q_{3}+\ldots+2^{r-2} q_{r}\right): \\
A_{1}=\left\langle\left(\begin{array}{c}
1 \\
-1 \\
0 \\
0 \\
0 \\
\vdots \\
0
\end{array}\right),\left(\begin{array}{c}
0 \\
0 \\
1 \\
-1 \\
0 \\
\vdots \\
0
\end{array}\right), \ldots,\left(\begin{array}{c}
0 \\
\vdots \\
0 \\
0 \\
0 \\
1 \\
-1
\end{array}\right)\right\rangle ;
\end{array}
$$$$
\lambda_{2}=-2^{2}\left(q_{2}+q_{3}+2 q_{4}+\ldots+2^{r-3} q_{r}\right) \text { : }
$$

$$
A_{2}=\left\langle\left(\begin{array}{c}
1 \\
1 \\
-1 \\
-1 \\
0 \\
0 \\
0 \\
0 \\
0 \\
\vdots \\
0
\end{array}\right),\left(\begin{array}{c}
0 \\
0 \\
0 \\
0 \\
1 \\
1 \\
-1 \\
-1 \\
0 \\
\vdots \\
0
\end{array}\right), \ldots,\left(\begin{array}{c}
0 \\
0 \\
0 \\
0 \\
0 \\
\vdots \\
0 \\
1 \\
1 \\
-1 \\
-1
\end{array}\right)\right\rangle \text {; }
$$




$$
\lambda_{r}=-2^{r} q_{r}: \quad A_{r}=\left\langle\left(\begin{array}{c}
1 \\
1 \\
\vdots \\
1 \\
-1 \\
-1 \\
\vdots \\
-1
\end{array}\right)\right\rangle \text {. }
$$

Разлагая начальное условие по собственным подпространствам $A_{\gamma}$, получаем решение задачи Коши:

$$
\begin{gathered}
\mathbf{f}(0)=\mathbf{a}_{0}+\mathbf{a}_{1}+\cdots+\mathbf{a}_{r}, \quad \mathbf{a}_{\gamma} \in A_{\gamma}, \\
\mathbf{f}(t)=\mathbf{a}_{0} e^{\lambda_{0} t}+\mathbf{a}_{1} e^{\lambda_{1} t}+\cdots+\mathbf{a}_{r} e^{\lambda_{r} t} .
\end{gathered}
$$

Отсюда видно, что стационарное решение представляет собой собственный вектор $\mathbf{a}_{0}$, соответствующий $\lambda_{0}=0$.

Отметим, что эта задача была также описана в терминах $p$-адического анализа в [26]. Задачу, рассмотренную в работе [25], нетрудно обобщить на случай дерева с произвольным индексом ветвления $m$. В этом случае система имеет $m^{r}$ состояний, а матрица переходов $A$ принимает следующий вид:

\begin{tabular}{|c|ccc|ccccccccc|c|}
\hline$d$ & $q_{1}$ & $\cdots$ & $q_{1}$ & $q_{2}$ & $q_{2}$ & $\cdots$ & $q_{2}$ & $\cdots$ & $q_{2}$ & $q_{2}$ & $\cdots$ & $q_{2}$ & $\cdots$ \\
\hline$q_{1}$ & $d$ & $\cdots$ & $q_{1}$ & $q_{2}$ & $q_{2}$ & $\cdots$ & $q_{2}$ & $\cdots$ & $q_{2}$ & $q_{2}$ & $\cdots$ & $q_{2}$ & $\cdots$ \\
$\vdots$ & $\vdots$ & $\ddots$ & $\vdots$ & $\vdots$ & $\vdots$ & $\cdots$ & $\vdots$ & $\cdots$ & $\vdots$ & $\vdots$ & $\cdots$ & $\vdots$ & $\cdots$ \\
$q_{1}$ & $q_{1}$ & $\cdots$ & $d$ & $q_{2}$ & $q_{2}$ & $\cdots$ & $q_{2}$ & $\cdots$ & $q_{2}$ & $q_{2}$ & $\cdots$ & $q_{2}$ & $\cdots$ \\
\hline$q_{2}$ & $q_{2}$ & $\cdots$ & $q_{2}$ & $d$ & $q_{1}$ & $\cdots$ & $q_{1}$ & $\cdots$ & $q_{2}$ & $q_{2}$ & $\cdots$ & $q_{2}$ & $\cdots$ \\
$q_{2}$ & $q_{2}$ & $\cdots$ & $q_{2}$ & $q_{1}$ & $d$ & $\cdots$ & $q_{1}$ & $\cdots$ & $q_{2}$ & $q_{2}$ & $\cdots$ & $q_{2}$ & $\cdots$ \\
$\vdots$ & $\vdots$ & $\vdots$ & $\vdots$ & $\vdots$ & $\vdots$ & $\ddots$ & $\vdots$ & $\cdots$ & $\vdots$ & $\vdots$ & $\cdots$ & $\vdots$ & $\cdots$ \\
$q_{2}$ & $q_{2}$ & $\cdots$ & $q_{2}$ & $q_{1}$ & $q_{1}$ & $\cdots$ & $d$ & $\cdots$ & $q_{2}$ & $q_{2}$ & $\cdots$ & $q_{2}$ & $\cdots$ \\
$\vdots$ & $\vdots$ & $\vdots$ & $\vdots$ & $\vdots$ & $\vdots$ & $\vdots$ & $\vdots$ & $\ddots$ & $\vdots$ & $\vdots$ & $\vdots$ & $\vdots$ & $\cdots$ \\
$q_{2}$ & $q_{2}$ & $\cdots$ & $q_{2}$ & $q_{2}$ & $q_{2}$ & $\cdots$ & $q_{2}$ & $\cdots$ & $d$ & $q_{1}$ & $\cdots$ & $q_{1}$ & $\cdots$ \\
$q_{2}$ & $q_{2}$ & $\cdots$ & $q_{2}$ & $q_{2}$ & $q_{2}$ & $\cdots$ & $q_{2}$ & $\cdots$ & $q_{1}$ & $d$ & $\cdots$ & $q_{1}$ & $\cdots$ \\
$\vdots$ & $\vdots$ & $\vdots$ & $\vdots$ & $\vdots$ & $\vdots$ & $\vdots$ & $\vdots$ & $\cdots$ & $\vdots$ & $\vdots$ & $\ddots$ & $\vdots$ & $\cdots$ \\
$q_{2}$ & $q_{2}$ & $\cdots$ & $q_{2}$ & $q_{2}$ & $q_{2}$ & $\cdots$ & $q_{2}$ & $\cdots$ & $q_{1}$ & $q_{1}$ & $\cdots$ & $d$ & $\cdots$ \\
\cline { 1 - 1 } & $\vdots$ & $\vdots$ & $\vdots$ & $\vdots$ & $\vdots$ & $\vdots$ & $\vdots$ & $\vdots$ & $\vdots$ & $\vdots$ & $\vdots$ & $\vdots$ & $\ddots$ \\
\hline
\end{tabular}

Здесь $d=-(m-1)\left[q_{1}+m q_{2}+\ldots+m^{r-1} q_{r}\right]$. Эта матрица также имеет $r+1$ собственное подпространство, которые строятся аналогично. А именно, собственный вектор, соответствующий нулевому собственному значению, это вектор $(1,1, \ldots, 1)$. Далее, чтобы построить подпространство $A_{\gamma}$, следует разбить вектор на блоки длины $m^{\gamma}$, выбрать один из них, а остальные заполнить нулями. В выбранном блоке необходимо выделить $m$ подблоков длины $m^{\gamma-1}$, каждый $i$-тый из них заполнить числом $x_{i}$, так чтобы $\sum_{i=1}^{m} x_{i}=0$. 
Таким способом, можно построить $(m-1) m^{r-\gamma}$ линейно независимых векторов, порождающих собственное подпространство $A_{\gamma}$ с собственным значением

$$
\lambda_{\gamma}=-m^{\gamma} q_{\gamma}-(m-1) m^{\gamma}\left(q_{\gamma+1}+m q_{\gamma+2}+\ldots+m^{r-\gamma-1} q_{r}\right)
$$

Аналогично, получаем решение задачи Коши. (Описание этой задачи в терминах $m$-адического анализа см. в [27].)

Следует отметить, что ультраметрическое случайное блуждание с симметричной матрицей переходов соотносится только с весьма узким классом физических систем, у которых все состояния энергетически вырождены. В ряде случаев, как, например, при спектральной диффузии в низкотемпературных белках [8], ультраметрическая диффузия на сильно вырожденном энергетическом ландшафте хорошо описывает наблюдаемые свойства. Однако это скорее счастливое исключение, чем правило. Гораздо чаще энергетические состояния макро- и супрамолекулярных структур не вырождены и имеют хорошо определенное основное состояние (глобальный минимум) с большим бассейном притяжения (см., например, $[28,29])$. Динамику таких систем тоже можно пытаться описывать ультраметрическим случайным блужданием при том понимании, что в невырожденном случае вся древообразная структура активационных барьеров помещена в «охватывающий» потенциал, выделяющий бассейн притяжения. Хотя уравнения для таких систем (в $p$-адической форме) обсуждались, например, в [30-32], существенного продвижения в части методов их решения и получения самих решений добиться пока не удалось. В данной статье мы рассматриваем уравнение для задачи с определённым видом потенциала, а именно, потенциальной ямой с одним основным состоянием, и показываем, что в предельном случае сильного поля удается найти точное аналитическое решение соответствующей задачи Коши.

Ультраметрическое блуждание в центрально-симметричном поле. Видоизменим описанное выше симметричное дерево переходов, поместив его в потенциальную яму с минимумом энергии в нулевом состоянии, которое далее будем называть основным состоянием системы (см. рис. 2). Назовём уровнем $\gamma(i)$ состояния $i$ ультраметрическое расстояние до основного состояния. Обозначим высоту состояния уровня $\gamma$ относительно нулевого уровня за $h_{\gamma}$, а высоту переходной вершины между состояниями $i$ и $j$ за $H_{i j}$. Тогда барьер перехода из $i$ в $j$ есть $H_{i j}-h_{\gamma(i)}$. Положим $H_{\gamma(i)}=H_{0 i}$, тогда константа

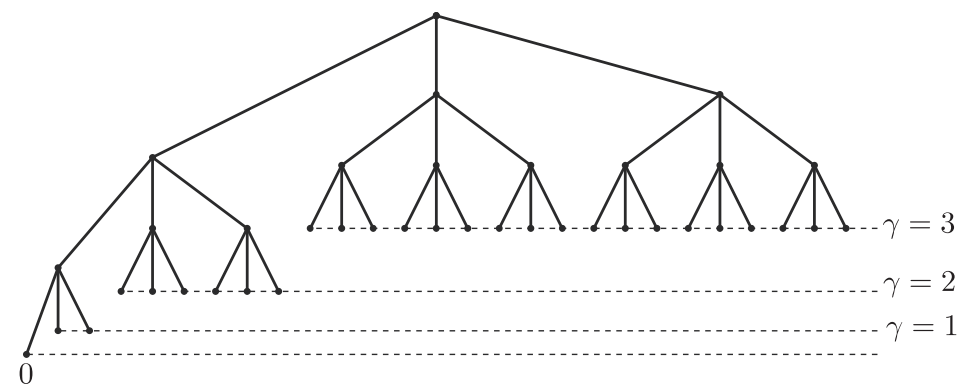

Рис. 2. Нерегулярное дерево при $m=3, r=3$ [Figure 2. Irregular tree, when $m=3, r=3$ ] 
перехода $a_{\gamma}$ из основного состояния в состояние уровня $\gamma$

$$
a_{\gamma}=\frac{e^{-H_{\gamma}}}{(m-1) m^{\gamma-1}}
$$

Заметим, что барьеры переходов между состояниями одного уровня не меняются по отношению к задаче с симметричным деревом, поэтому для переходов, не увеличивающих уровень состояния, соответствующие константы остаются прежними и имеют вид

$$
q_{\gamma}=\frac{e^{-\left(H_{\gamma}-h_{\gamma}\right)}}{(m-1) m^{\gamma-1}}
$$

Вычислим константы переходов для переходов $i \rightarrow j$, увеличивающих уровень состояния:

$$
q_{i \rightarrow j}=\frac{e^{-\left(H_{i j}-h_{\gamma(i)}\right)}}{(m-1) m^{\gamma(i)-1}}=\frac{e^{-H_{\gamma(j)}}}{(m-1) m^{\gamma(i)-1}} e^{h_{\gamma(i)}}=a_{j} s_{i}, \quad \text { где } \quad s_{i}=e^{h_{\gamma(i)}} .
$$

\begin{tabular}{|c|c|c|c|c|c|c|c|c|c|c|c|c|}
\hline$d_{0}$ & $q_{1}$ & $\cdots$ & $q_{1}$ & $q_{2}$ & $\cdots$ & $q_{2}$ & $\cdots$ & $q_{2}$ & $\cdots$ & $q_{2}$ & $q_{3}$ & $\cdots$ \\
\hline$a_{1}$ & $d_{1}$ & $\cdots$ & $q_{1}$ & $q_{2}$ & $\ldots$ & $q_{2}$ & $\ldots$ & $q_{2}$ & $\ldots$ & $q_{2}$ & $q_{3}$ & $\cdots$ \\
\hline$\vdots$ & $\vdots$ & $\ddots$ & $\vdots$ & $\vdots$ & $\ldots$ & $\vdots$ & $\ldots$ & $\vdots$ & $\ldots$ & $\vdots$ & $\vdots$ & $\ldots$ \\
\hline$a_{1}$ & $q_{1}$ & $\cdots$ & $d_{1}$ & $q_{2}$ & $\cdots$ & $q_{2}$ & $\cdots$ & $q_{2}$ & $\ldots$ & $q_{2}$ & $q_{3}$ & $\cdots$ \\
\hline$a_{2}$ & $a_{2} s_{1}$ & $\cdots$ & $a_{2} s_{1}$ & $d_{2}$ & $\cdots$ & $q_{1}$ & $\cdots$ & $q_{2}$ & $\cdots$ & $q_{2}$ & $q_{3}$ & $\cdots$ \\
\hline$\vdots$ & $\vdots$ & $\vdots$ & $\vdots$ & : & $\ddots$ & : & $\ldots$ & : & $\ldots$ & $\vdots$ & $\vdots$ & . . \\
\hline$a_{2}$ & $a_{2} s_{1}$ & $\cdots$ & $a_{2} s_{1}$ & $q_{1}$ & $\cdots$ & $d_{2}$ & $\ldots$ & $q_{2}$ & $\ldots$ & $q_{2}$ & $q_{3}$ & $\cdots$ \\
\hline$\vdots$ & $\vdots$ & $\vdots$ & $\vdots$ & : & $\vdots$ & : & $\ddots$ & $\vdots$ & $\vdots$ & $\vdots$ & $\vdots$ & $\cdots$ \\
\hline$a_{2}$ & $a_{2} s_{1}$ & $\cdots$ & $a_{2} s_{1}$ & $q_{2}$ & $\cdots$ & $q_{2}$ & $\cdots$ & $d_{2}$ & $\cdots$ & $q_{1}$ & $q_{3}$ & $\cdots$ \\
\hline$\vdots$ & $\vdots$ & $\vdots$ & : & $\vdots$ & : & $\vdots$ & $\ldots$ & : & $\ddots$ & $\vdots$ & $\vdots$ & 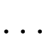 \\
\hline$a_{2}$ & $a_{2} s_{1}$ & $\ldots$ & $a_{2} s_{1}$ & $q_{2}$ & $\ldots$ & $q_{2}$ & $\ldots$ & $q_{1}$ & $\ldots$ & $d_{2}$ & $q_{3}$ & $\cdots$ \\
\hline$a_{3}$ & $a_{3} s_{1}$ & $\cdots$ & $a_{3} s_{1}$ & $a_{3} s_{2}$ & $\cdots$ & $a_{3} s_{2}$ & $\cdots$ & $a_{3} s_{2}$ & $\cdots$ & $a_{3} s_{2}$ & $d_{3}$ & $\cdots$ \\
\hline$\vdots$ & $\vdots$ & $\vdots$ & $\vdots$ & : & $\vdots$ & $\vdots$ & $\vdots$ & $\vdots$ & $\vdots$ & $\vdots$ & $\vdots$ & \\
\hline
\end{tabular}

Запишем матрицу переходов:

Здесь также $d_{0}, d_{1}, \ldots, d_{r}$ - суммы недиагональных элементов по соответствующим столбцам, взятые со знаком минус.

Построим собственные векторы такой матрицы. Заметим, что матрица (3) имеет блочно-иерархическую структуру, аналогичную матрице (2), поэтому каждый собственный вектор матрицы (3) можно также разбить на блоки, кратные индексу ветвления $m$. Более того, некоторые собственные векторы матрицы (3) совпадают с собственными векторами матрицы (2). А именно, это векторы, содержащие нули в первом блоке. Легко найти и некоторые другие векторы следующим образом.

Возьмём первый блок размера $m^{k}$, разобьём его на $m$ подблоков размера $m^{k-1}$ и первый из них заполним нулями. Остальные $m-1$-числами $x_{i}$, 
$i=2, \ldots, m$, соответственно, так что $\sum_{i=2}^{m} x_{i}=0$. Как нетрудно убедиться, эти векторы также будут собственными для матрицы (3).

Остаётся ещё $r+1$ собственный вектор. В силу введённого в задачу потенциала эти собственные векторы отвечают переходам из основного состояния системы. Поскольку переходы $0 \rightarrow i$ зависят только от $\gamma(i)$, объединим состояния, находящиеся на одном уровне, и построим матрицу переходов для вектора заселённостей объединённых состояний, который также будем обозначать f. Для этого сначала сложим строки матрицы (3), имеющие одинаковые элементы в первом столбце, заменяя соответствующие блоки строк строкой-суммой, а затем удалим повторяющиеся столбцы. В результате получим матрицу $\tilde{A}$ размерности $(r+1) \times(r+1)$ :

$$
\left(\begin{array}{ccccc}
d_{0}^{\prime} & q_{1} & q_{2} & \cdots & q_{r} \\
(m-1) a_{1} & d_{1}^{\prime} & (m-1) q_{2} & \cdots & (m-1) q_{r} \\
(m-1) m a_{2} & (m-1) m a_{2} s_{1} & d_{2}^{\prime} & \cdots & (m-1) m q_{r} \\
\vdots & \vdots & \vdots & \ddots & \vdots \\
(m-1) m^{r-1} a_{r} & (m-1) m^{r-1} a_{r} s_{1} & (m-1) m^{r-1} a_{r} s_{2} & \ldots & d_{r}^{\prime}
\end{array}\right) .
$$

В этой матрице также сумма элементов по столбцам равна 0. Таким образом, задача сводится к нахождению собственных векторов и собственных значений матрицы (4). Ниже мы рассмотрим один физически интересный предельный случай, для которого такая задача может быть решена аналитически.

Случай сильного поля. Рассмотрим случай, когда запрещены переходы, увеличивающие уровень состояния. Это означает, что $a_{1}=a_{2}=\ldots=a_{r}=0$. В этом случае матрица $\tilde{A}$ примет вид

$$
\left(\begin{array}{cccccc}
0 & q_{1} & q_{2} & q_{3} & \ldots & q_{r} \\
0 & -q_{1} & (m-1) q_{2} & (m-1) q_{3} & \ldots & (m-1) q_{r} \\
0 & 0 & -m q_{2} & (m-1) m q_{3} & \ldots & (m-1) m q_{r} \\
0 & 0 & 0 & -m^{2} q_{3} & \ldots & (m-1) m^{2} q_{r} \\
\vdots & \vdots & \vdots & \vdots & \ddots & \vdots \\
0 & 0 & 0 & 0 & \ldots & -m^{r-1} q_{r}
\end{array}\right) .
$$

В такой задаче разумно взять начальное условие $\mathbf{f}_{0}=(0, \ldots, 0,1)$ и исследовать кинетику заселённости основного состояния (т. е. первую координату вектора $\mathbf{f}$ ).

Матрица (5) треугольная, поэтому её диагональные элементы есть собственные значения. Собственный вектор, соответсвующий $\lambda_{0}=0$, находится сразу:

$$
\mathbf{v}_{0}=(1,0, \ldots, 0)
$$

Рассматривая структуру матрицы (5), нетрудно понять, что достаточно вычислить собственный вектор $\mathbf{v}_{r}$, соответствующий $\lambda_{r}=-m^{r-1} q_{r}$, т. е. найти ненулевое решение матричного уравнения

$$
\frac{\partial \mathbf{v}_{r}}{\partial t}=\left[\tilde{A}-\lambda_{r} E\right] \mathbf{v}_{r}
$$


Остальные векторы $\mathbf{v}_{k}$ находятся аналогичным образом заменой $r=k$ и заполнением координат $x_{i}, i>k$, собственного вектора нулями.

Решение уравнения (6) находится последовательно методом Гаусса:

$$
\begin{gathered}
x_{r}=1, \\
x_{r-1}=-\frac{(m-1) q_{r}}{m q_{r}-q_{r-1}}, \\
x_{r-2}=-\frac{m(m-1) q_{r}\left(q_{r}-q_{r-1}\right)}{\left(m q_{r}-q_{r-1}\right)\left(m^{2} q_{r}-q_{r-2}\right)},
\end{gathered}
$$

Докажем, что при $k \neq 0$

$$
x_{r-k}=-\frac{m^{k-1}(m-1) q_{r}\left(q_{r}-q_{r-1}\right)\left(m q_{r}-q_{r-2}\right) \ldots\left(m^{k-2} q_{r}-q_{r-k+1}\right)}{\left(m q_{r}-q_{r-1}\right)\left(m^{2} q_{r}-q_{r-2}\right) \ldots\left(m^{k} q_{r}-q_{r-k}\right)} .
$$

Из соответствующей строки матрицы следует

$$
x_{r-k}=-\frac{m^{r-k-1}(m-1)\left[q_{r} x_{r}+q_{r-1} x_{r-1}+\ldots+q_{r-k+1} x_{r-k+1}\right]}{m^{r-1} q_{r}-m^{r-k-1} q_{r-k}} .
$$

Вычислим последовательно сумму в квадратных скобках.

$$
\begin{gathered}
q_{r} x_{r}+q_{r-1} x_{r-1}=\frac{m q_{r}\left(q_{r}-q_{r-1}\right)}{m q_{r}-q_{r-1}}, \\
q_{r} x_{r}+q_{r-1} x_{r-1}+q_{r-2} x_{r-2}=\frac{m^{2} q_{r}\left(q_{r}-q_{r-1}\right)\left(m q_{r}-q_{r-2}\right)}{\left(m q_{r}-q_{r-1}\right)\left(m^{2} q_{r}-q_{r-2}\right)}
\end{gathered}
$$

По индукции получим:

$$
q_{r} x_{r}+\ldots+q_{r-k+1} x_{r-k+1}=\frac{m^{k-1} q_{r}\left(q_{r}-q_{r-1}\right) \ldots\left(m^{k-2} q_{r}-q_{r-k+1}\right)}{\left(m q_{r}-q_{r-1}\right) \ldots\left(m^{k-1} q_{r}-q_{r-k+1}\right)}
$$

Подставляя сумму в выражение (7), получим $x_{r-k}$. Аналогично можно вывести формулу для $x_{0}$ :

$$
x_{0}=-\frac{\left(q_{r}-q_{r-1}\right)\left(m q_{r}-q_{r-2}\right) \ldots\left(m^{r-2} q_{r}-q_{1}\right)}{\left(m q_{r}-q_{r-1}\right)\left(m^{2} q_{r}-q_{r-2}\right) \ldots\left(m^{r-1} q_{r}-q_{1}\right)} .
$$

Для решения задачи Коши остаётся разложить начальное условие по построенному базису размерности $r+1$, решив матричное уравнение

$$
B \mathbf{c}=\mathbf{f}_{0}
$$

относительно вектора коэффициентов разложения с, где $B$ - матрица, состоящая из столбцов $\mathbf{v}_{k}$. Это уравнение также решается методом Гаусса. 
При произвольном выборе величин $q_{i}$ для матрицы (5) затруднительно получить компактные выражения для вектора с, но их можно получить в случае линейного роста энергий $H_{\gamma}-h_{\gamma}$ от уровня $\gamma$.

Случай сильного линейного поля. Зададим барьеры переходов $H_{\gamma}-h_{\gamma}=$ $=\gamma$. Тогда матрица $\tilde{A}$ и собственный вектор $\mathbf{v}_{r}$ примут вид

$$
\begin{aligned}
& \left(\begin{array}{ccccccc}
0 & e^{-1} & \frac{1}{m} e^{-2} & \frac{1}{m^{2}} e^{-3} & \ldots & \frac{1}{m^{r-2}} e^{-(r-1)} & \frac{1}{m^{r-1}} e^{-r} \\
0 & -e^{-1} & \frac{m-1}{m} e^{-2} & \frac{m-1}{m^{2}} e^{-3} & \ldots & \frac{m-1}{m^{r-2}} e^{-(r-1)} & \frac{m-1}{m^{r-1}} e^{-r} \\
0 & 0 & -e^{-2} & \frac{m-1}{m} e^{-3} & \ldots & \frac{m-1}{m^{r-3}} e^{-(r-1)} & \frac{m-1}{m^{r-2}} e^{-r} \\
0 & 0 & 0 & -e^{-3} & \ldots & \frac{m-1}{m^{r-4}} e^{-(r-1)} & \frac{m-1}{m^{r-3}} e^{-r} \\
\vdots & \vdots & \vdots & \vdots & \ddots & \vdots & \vdots \\
0 & 0 & 0 & 0 & \ldots & -e^{-(r-1)} & \frac{m-1}{m} e^{-r} \\
0 & 0 & 0 & 0 & \ldots & 0 & -e^{-r}
\end{array}\right), \\
& \mathbf{v}_{r}=\left(\begin{array}{c}
-\frac{1}{m^{r-1}} \frac{\left(m e^{r-1}-1\right) \ldots(m e-1)}{\left(e^{r-1}-1\right) \ldots(e-1)} \\
\frac{m-1}{m^{r-1}} \frac{\left(m e^{r-2}-1\right) \ldots(m e-1)}{\left(e^{r-1}-1\right)\left(e^{r-2}-1\right) \ldots(e-1)} \\
\vdots \\
\frac{m-1}{m^{k}} \frac{\left(m e^{k-1}-1\right) \ldots(m e-1)}{\left(e^{k}-1\right)\left(e^{k-1}-1\right) \ldots(e-1)} \\
\vdots \\
\frac{m-1}{m^{3}} \frac{\left(m e^{2}-1\right)(m e-1)}{\left(e^{3}-1\right)\left(e^{2}-1\right)(e-1)} \\
\frac{m-1}{m^{2}} \frac{m e-1}{\left(e^{2}-1\right)(e-1)} \\
\frac{m-1}{m} \frac{1}{e-1} \\
1
\end{array}\right)
\end{aligned}
$$

Для получения окончательного решения задачи Коши разложим начальное условие по собственному базису. Запишем систему (8) в матричном виде и решим её:

$$
\left(\begin{array}{cccccc}
1 & -1 & -\frac{1}{m} \frac{m e-1}{e-1} & -\frac{1}{m^{2}} \frac{\left(m e^{2}-1\right)(m e-1)}{\left(e^{2}-1\right)(e-1)} & \ldots & -\frac{1}{m^{r-1}} \frac{\left(m e^{r-1}-1\right) \ldots(m e-1)}{\left(e^{r-1}-1\right) \ldots(e-1)} \\
0 & 1 & \frac{m-1}{m} \frac{1}{e-1} & \frac{m-1}{m^{2}} \frac{m e-1}{\left(e^{2}-1\right)(e-1)} & \ldots & \frac{m-1}{m^{r-1}} \frac{\left(m e^{r-2}-1\right) \ldots(m e-1)}{\left(e^{r-1}-1\right) \ldots(e-1)} \\
0 & 0 & 1 & \frac{m-1}{m} \frac{1}{e-1} & \ldots & \frac{m-1}{m^{r-2}} \frac{\left(m e^{r-3}-1\right) \ldots(m e-1)}{\left(e^{r-2}-1\right) \ldots(e-1)} \\
0 & 0 & 0 & 1 & \ldots & \frac{m-1}{m^{r-3}} \frac{\left(m e^{r-4}-1\right) \ldots(m e-1)}{\left(e^{r-3}-1\right) \ldots(e-1)} \\
\vdots & \vdots & \vdots & \vdots & \ddots & \vdots \\
0 & 0 & 0 & 0 & \ldots & \frac{m}{m} \frac{1}{e-1} \\
0 & 0 & 0 & 0 & \ldots & 1
\end{array}\right) \times
$$




$$
\times\left(\begin{array}{c}
a_{0} \\
a_{1} \\
a_{2} \\
a_{3} \\
\vdots \\
a_{r-1} \\
a_{r}
\end{array}\right)=\left(\begin{array}{c}
0 \\
0 \\
0 \\
0 \\
\vdots \\
0 \\
1
\end{array}\right) .
$$

Будем находить последовательно значения $a_{i}$ :

$$
a_{r}=1, \quad a_{r-1}=-\frac{m-1}{m} \frac{1}{e-1}, \quad a_{r-2}=-\frac{m-1}{m^{2}} \frac{e-m}{\left(e^{2}-1\right)(e-1)},
$$

Докажем, что для $k<r$

$$
a_{r-k}=-\frac{m-1}{m^{k}} \frac{\left(e^{k-1}-m\right) \ldots(e-m)}{\left(e^{k}-1\right)\left(e^{k-1}-1\right) \ldots(e-1)} .
$$

Предположим, что это верно для $a_{r-i}$ при всех $i<k$. Покажем, что равенство верно и для $i=k$. Из матрицы (9) находим:

$$
\begin{aligned}
a_{r-k}= & \frac{m-1}{m} \frac{1}{e-1} \cdot \frac{m-1}{m^{k-1}} \frac{\left(e^{k-2}-m\right) \ldots(e-m)}{\left(e^{k-1}-1\right)\left(e^{k-2}-1\right) \ldots(e-1)}+ \\
+ & \frac{m-1}{m^{2}} \frac{m e-1}{\left(e^{2}-1\right)(e-1)} \cdot \frac{m-1}{m^{k-2}} \frac{\left(e^{k-3}-m\right) \ldots(e-m)}{\left(e^{k-2}-1\right)\left(e^{k-3}-1\right) \ldots(e-1)}+ \\
& +\ldots+\frac{m-1}{m^{k-1}} \frac{\left(m e^{k-2}-1\right) \ldots(m e-1)}{\left(e^{k-1}-1\right)\left(e^{k-2}-1\right) \ldots(e-1)} \cdot \frac{m-1}{m} \frac{1}{e-1}- \\
& \quad-\frac{m-1}{m^{k}} \frac{\left(m e^{k-1}-1\right) \ldots(m e-1)}{\left(e^{k}-1\right)\left(e^{k-1}-1\right) \ldots(e-1)} .
\end{aligned}
$$

Дальнейшее доказательство является прямым следствие следующей леммы.

Лемма. Для любых $x \neq 1, m \in \mathbb{R} u k \in \mathbb{N}$ верно равенство

$$
\begin{gathered}
\frac{\left(m x^{k-1}-1\right) \ldots(m x-1)}{\left(x^{k}-1\right)\left(x^{k-1}-1\right) \ldots(x-1)}=\frac{\left(x^{k-1}-m\right) \ldots(x-m)}{\left(x^{k}-1\right)\left(x^{k-1}-1\right) \ldots(x-1)}+ \\
+(m-1) \frac{1}{x-1} \cdot \frac{\left(x^{k-2}-m\right) \ldots(x-m)}{\left(x^{k-1}-1\right)\left(x^{k-2}-1\right) \ldots(x-1)}+ \\
+(m-1) \frac{m x-1}{\left(x^{2}-1\right)(x-1)} \cdot \frac{\left(x^{k-3}-m\right) \ldots(x-m)}{\left(x^{k-2}-1\right)\left(x^{k-3}-1\right) \ldots(x-1)}+ \\
\quad+\ldots+(m-1) \frac{\left(m x^{k-2}-1\right) \ldots(m x-1)}{\left(x^{k-1}-1\right)\left(x^{k-2}-1\right) \ldots(x-1)} \cdot \frac{1}{x-1} .
\end{gathered}
$$

Д оказ ательст во. Докажем лемму по индукции. В правой части равенства - $k$ слагаемых, которые обозначим $b_{i}, 1 \leqslant i \leqslant k$. Сложим первые два слагаемых: 


$$
\begin{aligned}
& b_{1}+b_{2}= \frac{\left(x^{k-2}-m\right) \ldots(x-m)}{\left(x^{k-1}-1\right)\left(x^{k-2}-1\right) \ldots(x-1)}\left[\frac{x^{k-1}-m}{x^{k}-1}+\frac{m-1}{x-1}\right]= \\
&=\frac{\left(x^{k-2}-m\right) \ldots(x-m)}{\left(x^{k-1}-1\right)\left(x^{k-2}-1\right) \ldots(x-1)} \cdot \frac{\left(x^{k-1}-1\right)(m x-1)}{\left(x^{k}-1\right)(x-1)}= \\
& \quad=\frac{1}{x^{k}-1} \cdot \frac{m x-1}{x-1} \cdot \frac{\left(x^{k-2}-m\right) \ldots(x-m)}{\left(x^{k-2}-1\right) \ldots(x-1)} .
\end{aligned}
$$

Пусть для суммы первых $n$ слагаемых верна формула

$$
b_{1}+\ldots+b_{n}=\frac{1}{x^{k}-1} \cdot \frac{\left(m x^{n-1}-1\right) \ldots(m x-1)}{\left(x^{n-1}-1\right) \ldots(x-1)} \cdot \frac{\left(x^{k-n}-m\right) \ldots(x-m)}{\left(x^{k-n}-1\right) \ldots(x-1)},
$$

докажем её для $n+1$ слагаемых. Имеем

$$
\begin{aligned}
& b_{n+1}=(m-1) \frac{\left(m x^{n-1}-1\right) \ldots(m x-1)}{\left(x^{n}-1\right)\left(x^{n-1}-1\right) \ldots(x-1)} \cdot \frac{\left(x^{k-n-1}-m\right) \ldots(x-m)}{\left(x^{k-n}-1\right)\left(x^{k-n-1}-1\right) \ldots(x-1)} \\
& b_{1}+\ldots+b_{n+1}= \\
&= \frac{\left(m x^{n-1}-1\right) \ldots(m x-1)}{\left(x^{n-1}-1\right) \ldots(x-1)} \cdot \frac{\left(x^{k-n-1}-m\right) \ldots(x-m)}{\left(x^{k-n}-1\right)\left(x^{k-n-1}-1\right) \ldots(x-1)} \times \\
& \quad \times\left[\frac{x^{k-n}-m}{x^{k}-1}+\frac{m-1}{x^{n}-1}\right]= \\
&= \quad \times \frac{\left(x^{k-n}-1\right)\left(m x^{n}-1\right)}{\left(x^{n-1}-1\right) \ldots(m x-1)} \cdot \frac{\left(x^{k-n-1}-m\right) \ldots(x-m)}{\left(x^{k-n}-1\right)\left(x^{k-n-1}-1\right) \ldots(x-1)} \times \\
&=\frac{1}{x^{k}-1} \cdot \frac{\left(m x^{n}-1\right) \ldots(m x-1)}{\left(x^{n}-1\right) \ldots(x-1)} \cdot \frac{\left(x^{k-n-1}-m\right) \ldots(x-m)}{\left(x^{k-n-1}-1\right) \ldots(x-1)} .
\end{aligned}
$$

Теперь, полагая $n=k$, получим

$$
b_{1}+\ldots+b_{k}=\frac{\left(m x^{k-1}-1\right) \ldots(m x-1)}{\left(x^{k}-1\right)\left(x^{k-1}-1\right) \ldots(x-1)} .
$$

Что и требовалось доказать.

Итак, остаётся найти $a_{0}$. Складывая все строки матрицы (9), получим строку $(1,0, \ldots, 0)$ и единицу в столбце свободных членов. Отсюда $a_{0}=1$.

Теперь можно выписать решение задачи Коши в терминах заселённостей уровней:

$$
\mathbf{f}(t)=\left(\begin{array}{c}
1 \\
0 \\
0 \\
0 \\
\vdots \\
0
\end{array}\right)-\frac{m-1}{m^{r-1}} \frac{\left(e^{r-2}-m\right) \ldots(e-m)}{\left(e^{r-1}-1\right)\left(e^{r-2}-1\right) \ldots(e-1)}\left(\begin{array}{c}
-1 \\
1 \\
0 \\
0 \\
\vdots \\
0
\end{array}\right) e^{-e^{-1} t}-
$$




$$
\begin{aligned}
& -\frac{m-1}{m^{r-2}} \frac{\left(e^{r-3}-m\right) \ldots(e-m)}{\left(e^{r-2}-1\right)\left(e^{r-3}-1\right) \ldots(e-1)}\left(\begin{array}{c}
-\frac{1}{m} \frac{m e-1}{e-1} \\
\frac{m-1}{m} \frac{1}{e-1} \\
1 \\
0 \\
\vdots \\
0
\end{array}\right) e^{-e^{-2} t}- \\
& -\frac{m-1}{m^{r-3}} \frac{\left(e^{r-4}-m\right) \ldots(e-m)}{\left(e^{r-3}-1\right)\left(e^{r-4}-1\right) \ldots(e-1)}\left(\begin{array}{c}
-\frac{1}{m^{2}} \frac{\left(m e^{2}-1\right)(m e-1)}{\left(e^{2}-1\right)(e-1)} \\
\frac{m-1}{m^{2}} \frac{m e-1}{\left(e^{2}-1\right)(e-1)} \\
\frac{m-1}{m} \frac{1}{e-1} \\
1 \\
\vdots \\
0
\end{array}\right) e^{-e^{-3} t}-\ldots
\end{aligned}
$$

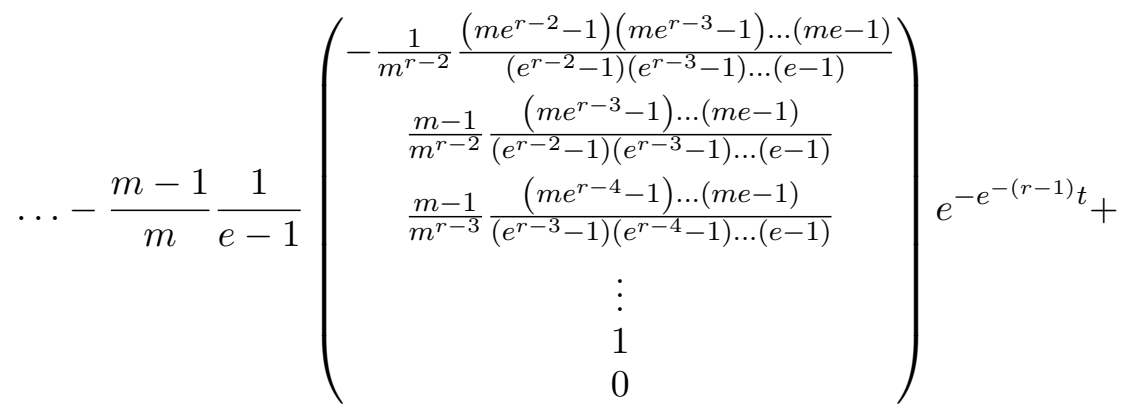

$$
\begin{aligned}
& +\left(\begin{array}{c}
-\frac{1}{m^{r-1}} \frac{\left(m e^{r-1}-1\right)\left(m e^{r-2}-1\right) \ldots(m e-1)}{\left(e^{r-1}-1\right)\left(e^{r-2}-1\right) \ldots(e-1)} \\
\frac{m-1}{m^{r-1}} \frac{\left(m e^{r-2}-1\right) \ldots(m e-1)}{\left(e^{r-1}-1\right)\left(e^{r-2}-1\right) \ldots(e-1)} \\
\frac{m-1}{m^{r-2}} \frac{\left(m e^{r-3}-1\right) \ldots(m e-1)}{\left(e^{r-2}-1\right)\left(e^{r-3}-1\right) \ldots(e-1)} \\
\vdots \\
\frac{m-1}{m} \frac{1}{e-1} \\
1
\end{array}\right) e^{-e^{-r} t} .
\end{aligned}
$$

Кинетика перехода в основное состояние. Рассмотрим первую координату вектора $\mathbf{f}(t)$ - зависимость заселённости основного состояния от времени, обозначив её $S(t)$ :

$$
\begin{aligned}
S(t)=1+ & \frac{m-1}{m^{r-1}}\left[\frac{\left(e^{r-2}-m\right) \ldots(e-m)}{\left(e^{r-1}-1\right)\left(e^{r-2}-1\right) \ldots(e-1)} \cdot e^{-e^{-1} t}+\right. \\
& +\frac{\left(e^{r-3}-m\right) \ldots(e-m)}{\left(e^{r-2}-1\right)\left(e^{r-3}-1\right) \ldots(e-1)} \frac{m e-1}{e-1} \cdot e^{-e^{-2} t}+\ldots
\end{aligned}
$$




$$
\begin{aligned}
\left.\ldots+\frac{1}{e-1} \frac{\left(m e^{r-2}-1\right)\left(m e^{r-3}-1\right) \ldots(m e-1)}{\left(e^{r-2}-1\right)\left(e^{r-3}-1\right) \ldots(e-1)} \cdot e^{-e^{-(r-1)} t}\right]- \\
\quad-\frac{1}{m^{r-1}} \frac{\left(m e^{r-1}-1\right)\left(m e^{r-2}-1\right) \ldots(m e-1)}{\left(e^{r-1}-1\right)\left(e^{r-2}-1\right) \ldots(e-1)} \cdot e^{-e^{-r} t} .
\end{aligned}
$$

При помощи численных расчётов в программе Wolfram Mathematica была построена асимптотика функции $S(t)$ на временном промежутке $\left(e^{1}, e^{r-1}\right)$. Как выяснилось,

$$
S(t) \sim C t^{a}
$$

где $a=\ln m+1$. Это означает, что в случае сильного поля, т.е. достаточно глубокого основного состояния, кинетика перехода имеет степенное поведение на промежутке характерных времён.

График поведения функции $S(t)$ при $m=3, r=15$ показан на рис. 3 , а на на рис. 4 изображён график $S(t)$ в логарифмической шкале, наложенный на график соответствующей степенной функции.

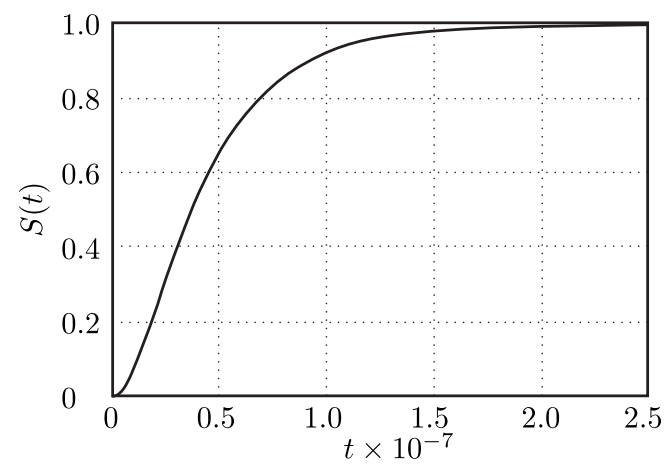

Рис. 3. График поведения релаксационной функции $S(t)$ при $m=3, r=15$ [Figure 3. The behaviour of the relaxation function $S(t)$, when $m=3, r=15]$

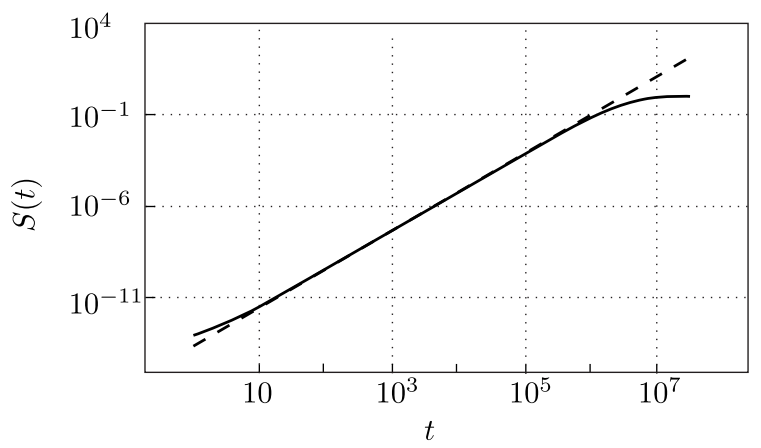

Рис. 4. График функции $S(t)$ (сплошная линия) в сравнении с функцией $\left(3 \cdot 10^{-14}\right) t^{\ln 3+1}$ (штриховая линия) в логарифмической шкале [Figure 4. A log-log plot of the function $S(t)$ (solid line) against the function $\left(3 \cdot 10^{-14}\right) t^{\ln 3+1}$ (dashed line)]

Заключение. Данный результат может представлять интерес для понимания особенностей кинетики коллапса полимерной цепи из клубкового состояния в глобулярное, в частности, для фолдинга белка. Действительно, следуя 
общепринятым представлениям (см., например, [33, с. 334], время перехода клубок-глобула можно характеризовать максимальным барьером, лежащим на пути перехода. Однако по тем же представлениям сам переход может пониматься как движение системы на многомерном ландшафте с сильно пересеченным энергетическим рельефом. Как показано выше, такие представления не противоречат друг другу, только если основное состояние достаточно глубокое.

Благодарности. Автор выражает благодарность Владику Аванесовичу Аветисову за обсуждение задачи и полезные замечания.

\section{ORCID}

Ольга Михайловна Сизова: http://orcid.org/0000-0002-7245-3071

\section{БИБЛИОГРАФИЧЕСКИЙ СПИСОК}

1. Сизова О. М. Ультраметрическая диффузия в сильном центрально-симметричном поле / Четвертая международная конферениия «Математическая физика и ее приложения»: материалы конф.; ред. чл.-корр. РАН И. В. Волович; д.ф.-м.н., проф. В. П. Радченко. Самара: СамГТУ, 2014. С. 329.

2. Владимиров В. С., Волович И. В., Зеленов Е. И. р-Адический анализ и математическая физика. М.: Наука, 1994. 352 с.

3. Хренников А. Ю., Шелкович В. М. Современный р-адический анализ и математическая физика: Теория и приложения. М.: Физматлит, 2012. 452 с.

4. Rammal R., Toulouse G., Virasoro M. A. Ultrametricity for phisicists // Rev. Mod. Phys., 1986. vol. 589, no. 3. pp. 765-788. doi : 10.1103/RevModPhys.58.765.

5. Mézard M., Parisi G., Virasoro M. A. Spin glass theory and beyond/ World Scientific Lecture Notes in Physics. vol. 9. Singapore: World Scientific, 1987. xiii+461 pp.

6. Доценко В. С. Физика спин-стекольного состояния // УФН, 1993. Т. 163, №6. С. 1-37. doi: 10.3367/UFNr.0163.199306a.0001.

7. Аветисов В. А., Бикулов А. Х., Зубарев А. П. Ультраметрическое случайное блуждание и динамика белковых молекул / Избранные вопросы математической физики и анализа: Сборник статей. K 90-летию со дня рождения академика Василия Сергеевича Владимирова/ Тр. МИАН, Т.285. М.: МАИК, 2014. С. 9-32. doi: 10.1134/ S0371968514020022.

8. Avetisov V., Bikulov A. Protein ultrametricity and spectral diffusion in deeply frozen proteins // Biophys. Rev. Lett., 2008. vol.03, no.03. pp. 387-396. doi:10.1142/ S1793048008000836.

9. Аветисов В. А., Бикулов А. Х., Зубарев А. П., Мешков Д. А. Многомасштабное математическое моделирование молекулярных машин: проблемы и современные подходы // Наноструктуры. Математическая физика и моделирование, 2011. Т. 6, № 1/2. С. 5-19.

10. Аветисов В. А., Бикулов А. Х., Зубарев А. П. О математическом моделировании молекулярных «нано-машин»// Вестн. Сам. гос. техн. ун-та. Сер. Физ.-мат. науки, 2011. T. 1(22). C. 9-15. doi: 10.14498/vsgtu906.

11. Аветисов В. А., Иванов А. Х., Мешков Д. А., Нечаев С. К. Фрактальная глобула как молекулярная машина // Писъмм в ЖЭТФ, 2013. Т. 98, № 4. С. 270-274. doi: 10.7868/ S0370274X13160121.

12. Avetisov V. A., Ivanov V. A., Meshkov D. A., Nechaev S. K. Fractal globules: a new approach to artificial molecular machines // Biophysical Journal, 2014. vol. 107, no. 4. pp. 2361-2368. doi: 10.1016/j.bpj.2014.10.019.

13. Mirny L. A. The fractal globule as a model of chromatin architecture in the cell// Chromosome Research, 2011. vol. 19, no. 1. pp. 37-51. doi: 10.1007/s10577-010-9177-0.

14. Nazarov L. I., Tamm M. V., Avetisov V. A., Nechaev S. K. A statistical model of intrachromosome contact maps // Soft Matter, 2015. vol. 11, no. 5. pp. 1019-1025. doi: 10.1039/ c4sm02519a ; arXiv: 1311.7689 [q-bio.GN]. 
15. Аветисов В. А., Журавлев Ю. Н. Эволюционная интерпретация -адического уравнения ультраметрической диффузии // Докл. РАН, 2007. Т. 414, № 3. С. 309-312.

16. Stillinger F. H., Weber Th. A. Hidden structure in liquids // Phys. Rev. A, 1982. vol. 25, no. 2. pp. 978-989. doi: 10.1103/PhysRevA.25.978.

17. Sherrington D. L. Landscape paradigms in physics and biology: Introduction and overview // Physica D: Nonlinear Phenomena, 1997. vol.107, no.2-4. pp. 117-121. doi:10.1016/ S0167-2789 (97)00076-6 ; arXiv: cond-mat/9608088.

18. Wright S. The role of mutation, inbreeding, crossbreeding and selection in evolution / Proceedings of the VI International Congress of Genetics; ed. D. Jones. Wisconsin: Brooklyn Botanic Garden, 1932. pp. 356-366.

19. Hoffmann K. H., Sibani P. Diffusion on hierarchies // Phys. Rev. A, 1988. vol.38, no. 8. pp. 4261-4270. doi : 10.1103/PhysRevA.38.4261.

20. Huberman B. A., Kerszberg M. Ultradiffusion: the relaxation of hierarchical systems // J. Phys. A: Math. Gen., 1985. vol. 18, no. 6. pp. L331-L335. doi: 10.1088/0305-4470/18/ $6 / 013$.

21. Blumen A., Klafter J., Zumofen G. Relaxation behaviour in ultrametric spaces // J. Phys. A: Math. Gen., 1986. vol. 19, no. 2. pp. L77-L84. doi: 10.1088/0305-4470/19/2/010.

22. Köhler G., Blumen A. Subordination on ultrametric spaces // J. Phys. A: Math. Gen., 1987. vol. 20, no. 16. pp. 5627-5634. doi: 10.1088/0305-4470/20/16/036.

23. Frauenfelder H. The connection between low-temperature kinetics and life/ Protein structure: Molecular and electronic reactivity. New York: Springer, 1987. pp. 245-261. doi: 10.1007/978-1-4612-4796-8_15.

24. Ansari A., Berendzen J., Bowne S. F., Frauenfelder H., Iben I. E., Sauke T. B., Shyamsunder E., Young R. D. Protein states and proteinquakes // Proc. Natl. Acad. Sci. USA, 1985. vol. 82, no. 15. pp. 5000-5004. doi: 10.1073/pnas.82.15.5000.

25. Ogielski A. T., Stein D. L. Dynamics on ultrametric spaces // Phys. Rev. Lett., 1985. vol. 55, no. 15. pp. 1634-1637. doi : 10.1103/PhysRevLett.55.1634.

26. Avetisov V. A., Bikulov A. H., Kozyrev S. V. Application of $p$-adic analysis to models of breaking of replica symmetry // J. Phys. A: Math. Gen., 1999. vol. 32, no. 50. pp. 8785-8791. doi : 10.1088/0305-4470/32/50/301; arXiv: cond-mat/9904360 [cond-mat.dis-nn].

27. Dolgopolov M. V., Zubarev A. P. Some aspects of $m$-adic analysis and its applications to $m$ adic stochastic processes // p-Adic Numbers, Ultrametric Analysis and Applications, 2011. vol. 3, no. 1. pp. 39-51. doi: 10.1134/S2070046611010043 ; arXiv: 1012.1248 [math-ph].

28. Becker O. M., Karplus M. The topology of multidimensional potential energy surfaces: Theory and application to peptide structure and kinetics // J. Chem. Phys., 1997. vol. 106, no. 4. pp. 1495-1517. doi: 10.1063/1.473299.

29. Wales D. J., Miller M. A., Walsh T. R. Archetypal energy landscapes // Nature, 1998. vol. 394. pp. $758-760$. doi : 10.1038/29487.

30. Kochubei A. N. Pseudo-differential equations and stochastics over non-Archimedean fields / Pure and Applied Mathematics. vol. 244. New York: Marcel Dekker, 2001. 336 pp.

31. Кочубей А. Н. Параболические уравнения над полем $p$-адических чисел// Изв. АН СССР. Сер. матем., 1991. Т. 55, № 6. С. 1312-1330.

32. Kozyrev S. V. Dynamics on rugged landscapes of energy and ultrametric diffusion // p-Adic Numbers, Ultrametric Analysis, and Applications, 2010. vol. 2, no. 2. pp. 122-132. doi: 10. $1134 / \mathrm{S} 2070046610020044$.

33. Гросберг А. Ю., Хохлов А. Р. Статистическал физика макромолекул. М.: Наука, 1988. $344 \mathrm{c.}$

Поступила в редакцию 16/XII/2014; в окончательном варианте - 15/II/2015; принята в печать $-25 / \mathrm{II} / 2015$. 
Vestn. Samar. Gos. Techn. Un-ta. Ser. Fiz.-mat. nauki

[J. Samara State Tech. Univ., Ser. Phys. \& Math. Sci.], 2015, vol. 19, no. 1, pp. 87-104

ISSN: 2310-7081 (online), 1991-8615 (print)

doi: http://dx.doi.org/10.14498/vsgtu1389

MSC: 15-02, 34L40, 60J27

ULTRAMETRIC DIFFUSION

IN A STRONG CENTRALLY SYMMETRIC FIELD*

\title{
O. M. Sizova
}

N. N. Semenov Institute of Chemical Physics,

Russian Academy of Sciences,

4, Kosygin st., Moscow, 119991, Russian Federation.

\begin{abstract}
A random process at the boundary of a finite regularly branching tree encapsulated in the central-symmetric external field is considered with respect to introduced ultrametricity. We demonstrate an explicit procedure of reduction of dimensionality of the problem. In addition, we consider the strongfield-limit and show that in this case the problem can be solved exactly. The exact solution of the strong-field-limit problem related to the case of linearly growing hierarchy of barriers is exemplified and supplemented by estimations of the transition kinetics into the ground state.
\end{abstract}

Keywords: ultrametricity, ultrametric diffusion, hierarchical energy landscape.

doi: http://dx.doi.org/10.14498/vsgtu1389

Acknowledgments. The author is grateful to Vladik A. Avetisov for helpful comments and insightful discussions..

\section{ORCID}

Olga M. Sizova: http://orcid.org/0000-0002-7245-3071

\section{REFERENCES}

1. Sizova O. M. Ultrametric diffusion in a strong centrally symmetric field, The 4nd International Conference "Mathematical Physics and its Applications", Book of Abstracts and Conference Materials; eds. I. V. Volovich; V. P. Radchenko. Samara, Samara State Technical Univ., 2014, pp. 329 (In Russian).

2. Vladimirov V. S., Volovich I. V., Zelenov E. I. p-Adic analysis and mathematical physics, Series on Soviet and East European Mathematics, vol. 1. Singapore, World Scientific, 1994, xviii+319 pp.. doi : 10.1142/9789814355933_fmatter.

(C) 2015 Samara State Technical University.

\section{How to cite Reference}

Sizova O. M. Ultrametric diffusion in a strong centrally symmetric field, Vestn. Samar. Gos. Tekhn. Univ., Ser. Fiz.-Mat. Nauki [J. Samara State Tech. Univ., Ser. Phys. \& Math. Sci.], 2015, vol. 19, no. 1, pp. 87-104. doi: 10.14498/vsgtu1389. (In Russian)

\section{Author Details}

Olga M. Sizova (olstet@mail.ru), Research Fellow, Dept. of Matter Structure.

${ }^{*}$ This paper is an extended version of the paper [1], presented at the Mathematical Physics and Its Applications 2014 Conference. 
3. Khrennikov A. Yu., Shelkovich V. M. Sovremennyi p-adicheskii analiz $i$ matematicheskaia fizika: Teoriia i prilozheniia [Modern $p$-adic analysis and mathematical physics: Theory and Applications]. Moscow, Fizmatlit, 2012, 452 pp. (In Russian)

4. Rammal R., Toulouse G., Virasoro M. A. Ultrametricity for phisicists, Rev. Mod. Phys., 1986, vol. 589, no. 3, pp. 765-788. doi: 10.1103/RevModPhys.58.765.

5. Mézard M., Parisi G., Virasoro M. A. Spin glass theory and beyond, World Scientific Lecture Notes in Physics, vol. 9. Singapore, World Scientific, 1987, xiii+461 pp.

6. Dotsenko V. S. Physics of the spin-glass state, Physics-Uspekhi, 1993, vol. 36, no. 6, pp. 455485. doi : 10.1070/pu1993v036n06abeh002161.

7. Avetisov V. A., Bikulov A. Kh., Zubarev A. P. Ultrametric random walk and dynamics of protein molecules, Proc. Steklov Inst. Math., 2014, vol. 285, pp. 3-25. doi: 10.1134/ S0081543814040026.

8. Avetisov V., Bikulov A. Protein ultrametricity and spectral diffusion in deeply frozen proteins, Biophys. Rev. Lett., 2008, vol.03, no.03, pp. 387-396. doi: 10.1142/ S1793048008000836.

9. Avetisov V. A., Bikulov A. Kh., Zubarev A. P., Meshkov D. A. Multiscale mathematical modeling of molecular machines: Problems and current approaches, Nanostruktury. Matematicheskaia fizika i modelirovanie, 2011, vol. 6, no. 1/2, pp. 5-19 (In Russian).

10. Avetisov V. A., Bikulov A. Kh., Zubarev A. P. Mathematical Modeling of Molecular "nanomachines", Vestn. Samar. Gos. Tekhn. Univ. Ser. Fiz.-Mat. Nauki [J. Samara State Tech. Univ., Ser. Phys. \& Math. Sci.], 2011, vol.1(22), pp. 9-15 (In Russian). doi: 10.14498/ vsgtu906.

11. Avetisov V. A., Ivanov V. A., Meshkov D. A., Nechaev S. K. Fractal globule as a molecular machine, JETP Letters, 2013, vol. 98, no. 4, pp. 242-246. doi : 10.1134/S0021364013170025.

12. Avetisov V. A., Ivanov V. A., Meshkov D. A., Nechaev S. K. Fractal globules: a new approach to artificial molecular machines, Biophysical Journal, 2014, vol. 107, no. 4, pp. 2361-2368. doi : 10.1016/j.bpj.2014.10.019.

13. Mirny L. A. The fractal globule as a model of chromatin architecture in the cell, Chromosome Research, 2011, vol. 19, no.1, pp. 37-51. doi : 10.1007/s10577-010-9177-0.

14. Nazarov L. I., Tamm M. V., Avetisov V. A., Nechaev S. K. A statistical model of intrachromosome contact maps, Soft Matter, 2015, vol.11, no. 5, pp. 1019-1025. doi: 10.1039/ c4sm02519a ; arXiv: 1311.7689 [q-bio.GN].

15. Avetisov V. A., Zhuravlev Yu. N. An evolutionary interpretation of the $p$-adic ultrametric diffusion equation, Dokl. Math., 2007, vol.75, no. 3, pp. 453-455. doi: 10.1134/ S1064562407030325.

16. Stillinger F. H., Weber Th. A. Hidden structure in liquids, Phys. Rev. A, 1982, vol. 25, no. 2, pp. 978-989. doi: 10.1103/PhysRevA.25.978.

17. Sherrington D. L. Landscape paradigms in physics and biology: Introduction and overview, Physica D: Nonlinear Phenomena, 1997, vol.107, no.2-4, pp. 117-121. doi: 10.1016/ S0167-2789 (97)00076-6 ; arXiv: cond-mat/9608088.

18. Wright S. The role of mutation, inbreeding, crossbreeding and selection in evolution, Proceedings of the VI International Congress of Genetics; ed. D. Jones. Wisconsin, Brooklyn Botanic Garden, 1932, pp. 356-366.

19. Hoffmann K. H., Sibani P. Diffusion on hierarchies, Phys. Rev. A, 1988, vol.38, no. 8, pp. 4261-4270. doi : 10.1103/PhysRevA.38.4261.

20. Huberman B. A., Kerszberg M. Ultradiffusion: the relaxation of hierarchical systems, J. Phys. A: Math. Gen., 1985, vol. 18, no. 6, pp. L331-L335. doi: 10.1088/0305-4470/18/ $6 / 013$.

21. Blumen A., Klafter J., Zumofen G. Relaxation behaviour in ultrametric spaces, J. Phys. A: Math. Gen., 1986, vol. 19, no. 2, pp. L77-L84. doi : 10.1088/0305-4470/19/2/010.

22. Köhler G., Blumen A. Subordination on ultrametric spaces, J. Phys. A: Math. Gen., 1987, vol. 20, no. 16, pp. 5627-5634. doi: 10.1088/0305-4470/20/16/036. 
23. Frauenfelder H. The connection between low-temperature kinetics and life, Protein structure: Molecular and electronic reactivity. New York, Springer, 1987, pp. 245-261. doi : 10.1007/978-1-4612-4796-8_15.

24. Ansari A., Berendzen J., Bowne S. F., Frauenfelder H., Iben I. E., Sauke T. B., Shyamsunder E., Young R. D. Protein states and proteinquakes, Proc. Natl. Acad. Sci. USA, 1985, vol. 82, no. 15, pp. 5000-5004. doi: 10.1073/pnas.82.15.5000.

25. Ogielski A. T., Stein D. L. Dynamics on ultrametric spaces, Phys. Rev. Lett., 1985, vol. 55, no. 15, pp. 1634-1637. doi: 10.1103/PhysRevLett.55.1634.

26. Avetisov V. A., Bikulov A. H., Kozyrev S. V. Application of $p$-adic analysis to models of breaking of replica symmetry, J. Phys. A: Math. Gen., 1999, vol. 32, no. 50, pp. 8785-8791. doi : 10.1088/0305-4470/32/50/301; arXiv: cond-mat/9904360 [cond-mat.dis-nn].

27. Dolgopolov M. V., Zubarev A. P. Some aspects of $m$-adic analysis and its applications to $m$-adic stochastic processes, $p$-Adic Numbers, Ultrametric Analysis and Applications, 2011, vol. 3, no. 1, pp. 39-51. doi: 10.1134/S2070046611010043; arXiv: 1012.1248 [math-ph].

28. Becker O. M., Karplus M. The topology of multidimensional potential energy surfaces: Theory and application to peptide structure and kinetics, J. Chem. Phys., 1997, vol. 106, no. 4, pp. 1495-1517. doi: 10.1063/1.473299.

29. Wales D. J., Miller M. A., Walsh T. R. Archetypal energy landscapes, Nature, 1998, vol. 394, pp. 758-760. doi : 10.1038/29487.

30. Kochubei A. N. Pseudo-differential equations and stochastics over non-Archimedean fields, Pure and Applied Mathematics, vol. 244. New York, Marcel Dekker, 2001, 336 pp.

31. Kochuber A. N. Parabolic equations over the field of $p$-adic numbers, Math. USSR-Izv., 1992, vol. 39, no. 3, pp. 1263-1280. doi: 10.1070/IM1992v039n03ABEH002247.

32. Kozyrev S. V. Dynamics on rugged landscapes of energy and ultrametric diffusion, $p$-Adic Numbers, Ultrametric Analysis, and Applications, 2010, vol. 2, no. 2, pp. 122-132. doi: 10. 1134/S2070046610020044.

33. Grosberg A. Yu., Khokhlov A. R. Statistical Physics of Macromolecules. New York, American Institute of Physics, 1994, 350 pp.

Received 16/XII/2014;

received in revised form 15/II/2015;

accepted 25/II/2015. 